\title{
Retropharyngeal Hematoma Secondary to Minor Blunt Neck Trauma: Case Report
}

\author{
Ahmet Can Senel ${ }^{1}$, Abdul Kadir Gunduz ${ }^{2}$
}

Summary: Senel AC, Gunduz AK - Retropharyngeal Hematoma Secondary to Minor Blunt Neck Trauma: Case Report.

Background and objectives: The development of a retropharyngeal hematoma following a blunt trauma is a rare occurrence. However, a spaceoccupying lesion in this area can be life-threatening and requires rapid assessment and treatment. This is clinically important because of the close proximity of the retropharyngeal space to the upper airway. Any swelling in the the posterior pharyngeal space may prompt it to bulge anteriorly into the airway and cause its obstruction.

Case Report: A 86-year-old woman fell from a height of $1.5 \mathrm{~m}$ and hit her neck on stairs and was admitted to our trauma department. A computerized tomography scan and a lateral neck x-ray to observe the soft tissue revealed a large retropharyngeal mass extending from the base of the skull down to the level of the lung. The patient was kept under observation and the hematoma reduced spontaneously in the following seven days although the patient died on the tenth day from pulmonary infection. The report emphasizes that early drainage of a retropharyngeal hematoma should be considered in similar cases. Although rarely encountered, retropharyngeal hematoma is a problem that can be faced in an emergency department.

Keywords: Pharyngeal Diseases; Hematoma; Airway obstruction; Dyspnea; Intubation, Intratracheal.

@2012 Elsevier Editora Ltda. All rights reserved.

\section{INTRODUCTION}

The development of a retropharyngeal hematoma following a blunt trauma is a rare occurrence. However, a space-occupying lesion in this area can be life-threatening and requires rapid assessment and treatment. There have been 63 cases of retropharyngeal hematoma reported in the English literature since $1966{ }^{1}$. They are clinically important because of the close proximity of the retropharyngeal space to the upper airway. Any swelling in the posterior pharyngeal space may prompt it to bulge anteriorly into the airway and cause its obstruction. Clinical management begins with securing the patient's airway. Diagnosis rests upon clinical examination and radiographic studies. Treatment depends upon the size of the hematoma as well as its clinical course. We report a case of retropharyngeal hematoma following minor blunt neck trauma. We review the literature and discuss management and treatment principles for this condition.

\footnotetext{
Received from Karadeniz Technical University Faculty of Medicine Department of Anesthesiology and Critical Care, Trabzon, Turkey.

1. MD; Associate Professor, Karadeniz Technical University, Faculty of Medicine, Department of Anesthesiology and Critical Care, Trabzon, Turkey.

2. MD; Associate Professor, Karadeniz Technical University, Faculty of Medicine, Department of Emergency, Trabzon, Turkey.

Submitted on April 12, 2012

Approved on June 12, 2012.

Corresponding Author:

Ahmet Can Senel, MD

Karadeniz Technical University, Faculty of Medicine

Department of Anesthesiology and Critical Care 61080 Trabzon, Turkey.

Telephone: 905323754785

E-mail: acsenel@gmail.com
}

\section{CASE REPORT}

A 86-year-old woman fell from a height of $1.5 \mathrm{~m}$ and hit her neck on the stairs. She was admitted to our trauma department transferred from a secondary hospital. The patient was first admitted to the secondary hospital emergency room with respiratory insufficiency. The patient was agitated and cyanosed. An orotracheal intubation was performed with difficulty due to pharyngeal deviation and compression as well as tracheal compression. After the patient stabilized she was transferred to our trauma department. On admission, the physical examination revealed vital signs with blood pressure (BP) at $90 / 40 \mathrm{~mm} \mathrm{Hg}$, Pulse of $95 \mathrm{bpm}$, and respiratory rate of 24 breathings per minute.

The neck was swollen and edematous, mainly in the left area. She was intubated orotracheal, and still agitated. There was no other pathological findings on physical examination. Neurological evaluation was normal and Glasgow Coma Score (GCS) revealed Eye opening: 4, Motor activity: 6, and Verbal response: 1 (Intubated). Arterial blood gas (ABG) values were $\mathrm{pH}: 7,447 ; \mathrm{PO}_{2}: 140.9 \mathrm{~mm} \mathrm{Hg}, \mathrm{pCO}_{2}: 31.2 \mathrm{~mm} \mathrm{Hg}$, $\mathrm{HCO}_{3}: 21.1 \mathrm{mEq} \cdot \mathrm{L}^{-1}$. Cell blood count $(\mathrm{CBC})$ analyses results were: Hemoglobin 9.7; White blood cell (WBC) 25,000; Thrombocytes 80,000; and Hematocrit 27.5. Biochemical analysis, Prothrombin time (PT) and partial Thromboplastin time (PTT) and bleeding time were normal. Cervical X-ray showed C4-C5 under a low density. Computed tomography (CT) scan of the neck revealed that at the level of C2 and left anterior border of the trachea there was a hypodense $3 x$ $1.5 \mathrm{~cm}$ area, a deviation of trachea to the right and a cannula inside it (Figure 1). 


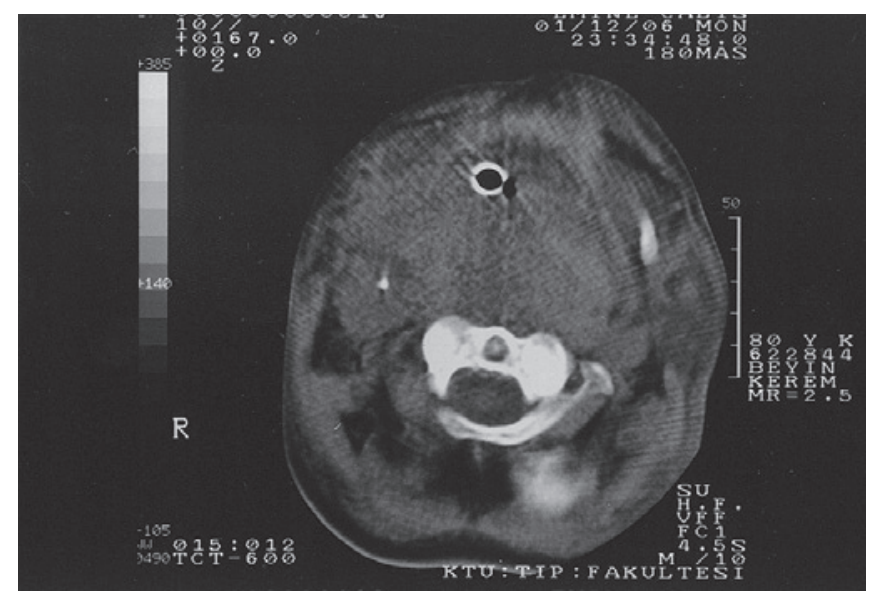

Figure 1 - Computerized Tomography of the Patient.

Ear, nose and throat (ENT) and intensive care consultations were done. Relying on the ABG and the patient's clinical evaluation, the ENT and emergency department surgeons decided to keep the patient under observation at the intensive care unit. Cervical immobilization with a hard collar (Philadelphia collar), antibiotics (ceftriaxone $100 \mathrm{mg} \cdot \mathrm{kg}^{-1}$ ), appropriate intravenous fluids (total: 2,500; $1,000 \mathrm{~mL}$ of isotonic (SF), $500 \mathrm{~mL}$ of Ringer Lactate, $1,000 \mathrm{~mL}$ of $5 \%$ Dextrose) and se-

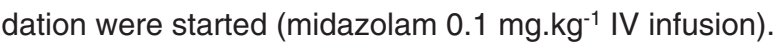

There was no evacuated material from the pharyngeal area. The hematoma and edema regressed during the follow up. A tracheotomy was performed in the fourth day of observation at Emergency Department (ED), considering complications of endotracheal intubation. However, the patient died from lung infection and multiple organ failure at the 10th day of observation despite appropriate intravenous antibiotics.

\section{DISCUSSION}

Retropharyngeal hematoma, although extremely uncommon, is a well known complication of cervical trauma, neck surgery, deep neck infections, foreign bodies, great vessel trauma or carotid aneurysm and hemorrhagic parathyroid adenoma. In addition, violent neck and body movements caused by coughing, vomiting or muscular exercise have also been reported as etiologies ${ }^{2}$. Retropharyngeal hematoma can occur spontaneously in patients with bleeding disorders ${ }^{3}$. Three cases were reported in patients taking anticoagulants ${ }^{4}$.

Hematoma formation in the retropharyngeal space is clinically important because of the potential risk for airway obstruction. Anatomically the space between the vertebral bodies and the pharyngeal muscles is divided into three potential spaces (the retropharyngeal space, the danger space, and prevertebral space) by three layers of fascia (the visceral fascia, the alar fascia, and the prevertebral fascia).
Our patient developed retropharyngeal hematoma after falling from a height of $1.5 \mathrm{~m}$ and hitting her neck at stairs. There was no additional trauma like cranial, thoracic, abdominal and of extremities. Cervical X-ray and cervical CT did not show a cervical dislocation and compression.

As with many head and neck pathologies, treatment of retropharyngeal hematoma starts with the maintenance and protection of the airway from obstruction. Many authors advocate tracheotomy as the procedure of choice for maintaining an airway. Some consider retropharyngeal hematoma as a contraindication to endotracheal intubation because of the potential for perforation of the retropharyngeal mass by the procedure ${ }^{5}$.

Once the airway is secured, two options are available: drainage or observation. Surgical exploration and transoral aspiration have been tried but showed no advantage over conservative treatment, and entailed an increased risk of infection ${ }^{1}$. Some authors advocate observation and prescribe drainage for those hematoma that do not reabsorb. These authors cite that many hematoma have been reported to decrease over a 2-3 week period. Patients with small, non-expanding hematoma can be treated conservatively with cervical spine immobilization ${ }^{3}$. For larger, as well as for those which fail to regress, drainage is indicated. Two routes of drainage are described; transoral aspiration and external drainage. Surgical drainage is essential for large hematoma especially for those rapidly expanding. In our case, no problems were faced in relation to securing the air passage of the patient. The patient's general condition was good and hematoma did not expand. There was no evacuated material from this area and the hematoma and edema regressed in the follow up, so surgery was not planned. The hematoma reduced spontaneously in the following seven days. However, patient died on the tenth day from pulmonary infection despite appropriate intravenous antibiotics.

We carried out a systematic analysis of the indexed articles published since 1977 in order to provide a better foundation for our study. We searched using the terms "hematoma" and "retropharyngeal" in the Lilacs (Literatura Latino-Americana e do Caribe em Ciências da Saúde), Pubmed (U.S. National Library of Medicine National Institutes of Health) and Embase (ExcerptaMedica). Of the 129 references found in Pubmed alone, only 11 case reports referred to retropharyngeal hematoma due to secondary trauma (Table I).

Table I - Analysis of the Indexed Articles Published since 1977 referred to "Retropharyngeal Hematoma due to Secondary Trauma"

\begin{tabular}{lc}
\hline & $\begin{array}{c}\text { "hematoma" and "retropharyngeal" } \\
\text { in } 129 \text { references }\end{array}$ \\
\hline Lilacs & none \\
Embase & none \\
Pubmed & 11 \\
\hline
\end{tabular}

Although rarely encountered, retropharyngeal hematoma is a serious problem that can be faced in an emergency department. 


\section{REFERENCES}

1. Van Velde R, Sars PR, Olsman JG, Van De Hoeven H - Traumatic retropharyngeal haematoma treated by embolization of the thyrocervical trunk. Eur J Emerg Med, 2002;9:159-161.

2. Daniello NJ, Goldstein SI - Retropharyngeal hematoma secondary to minor blunts head and neck trauma. Ear Nose Throat J, 1994;73:4143.

3. Mackenzie JW, Jellicoe JA - Acute upper airway obstruction. Spontaneous retropharyngeal haematoma in a patient with polycythaemia rubra vera. Anaesthesia, 1986;41:57-60.

4. Owens DE, Calcatessa TC, Aarstad RA - Retropharyngeal hematoma. A complication of therapy with anticoagulants. Arch Otolaryngol, 1975;101:565-568.

5. Senthuran S, Lim S, Gunning KE - Life-threatening airway obstruction caused by a retropharyngeal haematoma. Anaesthesia, 1999;54:674678. 